\title{
Hydrocortisone to treat early bronchopulmonary dysplasia in very preterm infants: study protocol for a randomized controlled trial
}

\author{
Yuan $\mathrm{He}^{1 \dagger}$, Yong Zhang ${ }^{2+}$, Shuqiang Gao ${ }^{3 \dagger}$, Xiaoling Wang ${ }^{1}$, Na He ${ }^{1}$, Deshuang Zhang ${ }^{1}$, Wenbin Dong ${ }^{1,4,5}$,
} Christian Wieg ${ }^{1,5,6^{*}}$ and Xiaoping Lei ${ }^{1,4,5^{*}}$ (D)

\begin{abstract}
Background: Bronchopulmonary dysplasia (BPD) is still a common complication in very premature infants. At present, there is no effective treatment for BPD. Glucocorticoids are drugs commonly used to prevent or treat BPD before and after birth. In very premature infants with high risk factors for BPD, early use of dexamethasone can reduce the rate of death and/or BPD but may cause long-term adverse neurodevelopmental outcomes. Hydrocortisone (HC), as an alternative drug to dexamethasone, has been increasingly used to prevent BPD. However, no study has reported the efficacy and safety of HC to treat early BPD diagnosed at postnatal day (PND) 28.
\end{abstract}

Methods: This study protocol is for a multicenter double-blind randomized controlled trial of low-dose HC in the treatment of early BPD. Early BPD infants will be randomly assigned to the $\mathrm{HC}$ treatment group or control group. Infants in the HC group will receive $0.5 \mathrm{mg} / \mathrm{kg} \mathrm{HC}$ twice a day for 7 days and then $0.5 \mathrm{mg} / \mathrm{kg} \mathrm{HC}$ once a day for 3 days. The control group will be given the same volume of placebo and no intervention on the basis of routine treatment. The primary outcome is survival without moderate or severe BPD at 36 weeks postmenstrual age. Secondary outcomes are the short- and long-term effects on growth, metabolism, neurodevelopment, and other possible complications.

Discussion: This trial will determine the efficacy and safety of low-dose $\mathrm{HC}$ administration compared to placebo for the reduction of moderate or severe BPD at 36 weeks postmenstrual age in very preterm infants with early BPD.

Trial registration: China Clinical Trial Registration Center ChiCTR1900021854. Registered on 13 March 2019.

Keywords: Bronchopulmonary dysplasia, Hydrocortisone, Preterm infants

\section{Background}

Bronchopulmonary dysplasia (BPD) is a serious complication in very preterm infants, and the incidence has been no significant decrease in the past two decades [1]. When BPD is present in preterm infants, mortality and

\footnotetext{
* Correspondence: Christian.Wieg@klinikum-ab-alz.de; leixiaopingde@126.com †Yuan He, Yong Zhang, and Shuqiang Gao contributed equally and are the co-first authors.

'Department of Neonatology, Affiliated Hospital of Southwest Medical University, 25 Taiping Road, Luzhou 646000, Sichuan, China Full list of author information is available at the end of the article
}

short- and long-term comorbidities are increased, and the need for health and education services is required more frequently than non-BPD infants later in life [2]. Since BPD was first described by Northway in 1967, the understanding of the pathophysiology evolved, and the definitions of BPD changed over time [3]. In 2001, the National Institute of Child Health and Human Development (NICHD) workshop defined BPD as the need for supplemental oxygen on the 28th day of life and categorized it as "mild, moderate, and severe" at 36th week

(c) The Author(s). 2020 Open Access This article is licensed under a Creative Commons Attribution 4.0 International License, which permits use, sharing, adaptation, distribution and reproduction in any medium or format, as long as you give appropriate credit to the original author(s) and the source, provide a link to the Creative Commons licence, and indicate if changes were made. The images or other third party material in this article are included in the article's Creative Commons licence, unless indicated otherwise in a credit line to the material. If material is not included in the article's Creative Commons licence and your intended use is not permitted by statutory regulation or exceeds the permitted use, you will need to obtain permission directly from the copyright holder. To view a copy of this licence, visit http://creativecommons.org/licenses/by/4.0/ The Creative Commons Public Domain Dedication waiver (http://creativecommons.org/publicdomain/zero/1.0/) applies to the data made available in this article, unless otherwise stated in a credit line to the data. 
postmenstrual age (PMA) (or 56 days of age for infants $>32$ weeks GA), based on oxygen use and/or respiratory support [4]. Although this definition is widely used, its prognostic value is limited due to its inconsistent correlation with long-term respiratory outcomes and the inability to classify infants dying from severe respiratory failure prior to 36 weeks of PMA [5]. Thus, the Vermont-Oxford Network modified the BPD definition to need for oxygen supplementation at 36th week PMA $[6,7]$, perceiving a high accuracy in predicting long-term respiratory outcomes but being unable to guide the early treatment of BPD.

Inflammation plays a key role in the pathogenesis of BPD by providing the rationale for treating BPD with glucocorticoids, which have potent anti-inflammatory effects $[8,9]$. A large number of studies have shown that glucocorticoids, especially dexamethasone, improve short-term pulmonary function in evolving BPD, but there is no evidence for beneficial long-term pulmonary outcomes. Since the reports by Shinwell et al. [10], concerns regarding adverse neurodevelopmental outcomes of premature infants exposed early to dexamethasone have increased $[11,12]$. Supported by a systematic review, the actual conclusion of this debate is that the short-term benefits of postnatal dexamethasone do not outweigh the long-term adverse effects. Thus, dexamethasone is recommended only for infants who cannot be weaned from mechanical ventilation more than 7 days after birth [11-13].

As an alternative glucocorticoid, hydrocortisone $(\mathrm{HC})$ has been considered to replace dexamethasone $[14,15]$. In newborn rats, $\mathrm{HC}$ had no detrimental effect on neurodevelopment [16], and infants exposed to $\mathrm{HC}$ in neonatal life had a higher median motor optimality score at the age of 3 months compared to those with dexamethasone exposure [17]. Furthermore, some studies have indicated that exposure to low-dose $\mathrm{HC}(1-2 \mathrm{mg} / \mathrm{kg} / \mathrm{d})$ leads to increased survival without BPD $[9,18-21]$ and does not increase adverse long-term neurodevelopmental sequelae [22-25]. However, these studies only used $\mathrm{HC}$ as a prophylactic drug (started at $\leq 7$ days or between 7 and 14 days after birth) $[9,18-26]$.

In current clinical practice, according to the NICHD criteria, $68-77 \%$ of very preterm infants are diagnosed with BPD at 28 days of life (early BPD), while approximately $60 \%$ of early BPD infants still need oxygen or respiratory support at a PMA of 36 weeks (moderate or severe BPD) [27-29]. Thus, it is essential to find an effective and safe drug to prevent early BPD from developing to moderate or severe BPD. However, based on the current recommendations, except those needing mechanical ventilation, very preterm infants will not be treated with glucocorticoids at this stage. Based on the use of early low-dose $\mathrm{HC}$ in infants with a high risk of
BPD in previous studies [9, 18-25], an RCT study is reasonable to evaluate the efficacy and safety of low-dose $\mathrm{HC}$ in treating early BPD.

\section{Study design and methods \\ Aim of the study}

To investigate the efficacy and safety of a low dose of $\mathrm{HC}$ in the treatment of early BPD, diagnosed at postnatal day (PND) 28 in preterm infants.

\section{Study design}

This multicenter double-blind randomized controlled trial will be performed at three centers in China. The enrollment period will be 1 year, and there will be a series of follow-up for every child until 2 years corrected age, which is the revised age from the 40th gestation week for a premature baby. The human research ethics committees at each participating center approved the trial, and an independent academic board will monitor the trial.

\section{Participants}

Inclusion criteria:

1) Gestational age $26^{+0}$ to $30^{+6}$ weeks and birth weight $<1500 \mathrm{~g}$; and

2) Diagnosed early BPD at day 28 , based on NICHD criteria [4] and never received any glucocorticoids after birth; and

3) One of the following conditions:

- Oxygen reduction test showing $\mathrm{SpO}_{2}<90 \%$ with $\mathrm{FiO}_{2}>0.3$ or

- Noninvasive ventilation support or nasal cannula with $\mathrm{FiO}_{2}>0.21$.

Exclusion criteria included any of the following conditions:

1) Invasive ventilation support needed on the 28 th day of life.

2) Congenital malformations affecting pulmonary function (e.g., congenital surfactant protein deficiencies, congenital diaphragmatic hernia) or increasing the risk of death or adverse neurodevelopmental outcome (e.g., congenital cerebral malformations, congenital hydrocephalus).

3) Chromosomal defects (e.g., trisomy 13, 18, 21).

4) Severe infection less than 7 days before randomization (late-onset sepsis/meningitis confirmed by positive blood culture; bacterial pneumonia, confirmed by positive tracheal culture; neonatal necrotizing enterocolitis). 


\section{Sample size calculation}

According to the data from the participating centers in the past 2 years and the data presented from the range of previous studies [27-29], the incidence of early BPD in infants with gestational age $26^{+0}$ to $30^{+6}$ weeks was $68-77 \%$, and $40 \%$ developed moderate or severe BPD. The effect of HC therapy was assumed to reduce moderate or severe BPD (the primary endpoint) to $25 \%$ or less (number needed to treat, NNT =5). Adopting a maximum significance level of $\alpha=0.05$ and a minimum test power of $80 \%$, the number of subjects per group will be $N=81$, which will be increased by $20 \%$ to compensate for potential dropouts during the follow-up, leading to 98 subjects per group (total $98 \times 2=196$ subjects).

\section{Trial medication and dosing}

Trial medication will be prepared and distributed according to Good Clinical Practice (GCP) and Good Manufacturing Practice (GMP) guidelines. Using a double-blinded, placebo-controlled design, infants will be allocated to either $\mathrm{HC}$ or placebo treatment.

The long-term adverse neurodevelopmental outcomes observed with dexamethasone use are associated with the accumulative dosage [30]. The optimal (cumulative) dose of $\mathrm{HC}$ is currently unknown. In one previous study, Baud et al. [21] found that an early ( $<7$ days) low dose of $\mathrm{HC}$ increased the rate of survival without BPD at 36 weeks PMA without adverse neurodevelopmental outcomes at 2 years of age [22, 24]. Based on these findings, we adopted this dosage in the present study. Infants randomly assigned to the $\mathrm{HC}$ group will receive $0.5 \mathrm{mg} / \mathrm{kg}$ $\mathrm{HC}$ twice per day for 7 days, followed by one dose of 0.5 $\mathrm{mg} / \mathrm{kg}$ per day for 3 days. This leads to a total duration of therapy of 10 days and a cumulative dose of $8.5 \mathrm{mg} / \mathrm{kg}$ HC. The control group will receive an equal volume of saline and will not receive any other intervention on the basis of routine treatment.

\section{Use of cointervention}

All randomized patients will be subjected to cointerventions, such as respiratory support and comedication, according to the guidelines and protocols of the participating centers. The participants will not be given other treatments outside of the trial interventions and cointerventions.

\section{Informed consent}

Professional researchers will carefully introduce the detailed procedures, probable benefits, and potential risks of the study to the guardian of the infants who meet the inclusion criteria. The infants' guardians will indicate their willingness to participate voluntarily by signing an informed consent form.

\section{Randomization}

Eligible patients will be randomized at day 28 after birth in each center after at least one guardian provides informed consent. A list of all eligible patients not included in the study will be provided from all centers throughout the time of enrollment. This trial will be protected from selection bias by using concealed, stratified, and blocked randomization. Randomization will be stratified according to gestational age stratum $\left(26^{+0}\right.$ to $28^{+6}$ weeks, and $29^{+0}$ to $30^{+6}$ weeks) in each study center. Multiple birth infants will be internally randomized. The randomization procedure will be generated by using Excel software by the GCP faculty at the participating hospitals, and it will be sealed in a sequentially numbered envelope to maintain the allocation concealment. According to the allocation sequence, drug packages will be hidden and labeled sequentially. Participants will receive packaged drug in the order of recruitment. The first dose of study medication will be administered within $1 \mathrm{~h}$ after randomization.

The complete random sequence will be kept by the primary researcher at the affiliated hospital of Southwest Medical University. Centers will individually have emergency envelopes that contain the treatment information for patients. Emergency envelopes can be unsealed only under special conditions.

\section{Outcomes}

\section{Primary outcome}

The primary outcome is survival without moderate or severe BPD at PMA 36 weeks, which is defined as the following criteria: no respiratory support, spontaneous breathing $\mathrm{FiO}_{2} 0.21$ for $\mathrm{SpO}_{2}$ 92\%.

\section{Secondary outcomes}

1) Amplitude-integrated electroencephalography $(\mathrm{aEEG})$. A series of aEEGs will be monitored once a week for all preterm infants after 30 weeks PMA. Sleep-wake cycles of aEEG can be classified as absent, mature, or immature according to HellstromWestas [31]. The PMA of mature sleep-wake cycles occurred will be used as one of the neurodevelopmental outcomes and be compared between two groups.

2) Intraventricular hemorrhage (IVH) > grade II and/ or periventricular leucomalacia (PVL). A series of cranial ultrasound scans will be performed regularly for all preterm infants, and these results will be extracted from the medical records after enrollment. IVH > grade II and/or PVL will be used as an adverse outcome to be compared between the groups [32]. 
3) Brain volume at 40 weeks PMA. Total brain volume will be detected by using the functional MRI of the brain Software Library for automatic segmentation of the T1/T2 weighted images. The mean/median total brain volumes will be compared between groups at 40 weeks.

4) Nosocomial infection, including cultured proven sepsis, meningitis, and pneumonia. Culture-proven infection is defined as a positive culture obtained from sputum, urine, blood, or cerebrospinal fluid of infants with clinical signs of infection, and pneumonia will be confirmed by abnormal lung examination (such X-ray, CT).

5) Neonatal necrotizing enterocolitis (NEC). Bell stage II or more and/or focal intestinal perforation diagnosed by X-ray after enrollment to hospital discharge [33].

6) Gastrointestinal bleeding diagnosed by the contents of the digestive tract.

7) Retinopathy of prematurity (ROP). A series of fundus examinations will be arranged after enrollment, and ROP that needs treatment in any stage will be used as an adverse outcome [34].

8) Anthropometric indices. Body length, head circumference, and body weight at 36 weeks of PMA.

9) Hyperglycemia. Hyperglycemia that needs to be treated with insulin (serum glucose levels $\geq 200 \mathrm{mg}$ / dl) after enrollment.

10) Hospitalization days.

\section{Long-term outcomes after hospital discharge}

1) Neurodevelopmental impairment at 104 weeks corrected age: The Bayley Scales of Infant and Toddler Development, Second Edition (BSID II) will be used in this trial.

a. Cognitive delay: defined as a cognitive score less than 80 .

b. Language delay: defined as a language score less than 80 .

c. Cerebral palsy and severity of cerebral palsy using the gross motor function classification system.

2) Hearing loss requiring hearing aids at 104 weeks corrected age.

3) Blindness at 104 weeks corrected age: vision loss with only form or shadow vision or no useful vision.

4) Number of readmissions in the first year since the first discharge home.

5) Weight, length, and head circumference at 40 weeks postmenstrual age and 12, 26, 52, and 104 weeks corrected age.
6) Premature birth-related mortality from enrollment to 104 weeks corrected age.

7) Behavioral problems (child behavior checklist) at 104 weeks corrected age. The scores on the checklist will be compared between groups.

\section{Clinical data collection}

At the time of randomization, baseline data will be collected from all included patients, including maternal and patient characteristics. Demographic data will include sex, birth weight, gestational age, 5-min Apgar score, surfactant therapy, prenatal corticosteroid use, premature rupture of the membrane before the onset of labor $(>18 \mathrm{~h})$, singleton/multiple birth, and mode of delivery. Population characteristics will be used to compare outcomes between the two groups.

The following clinical data will be collected: continuous positive airway pressure (CPAP)-assisted time, invasive ventilator-assisted time, days of hospitalization, days on supplemental oxygen, IVH, spontaneous intestinal perforation, NEC, and ROP.

During HC treatment, respiratory support, blood sugar, blood pressure, and anthropometric data will be collected. Outcome variables and cointerventions will be recorded at 36 weeks PMA.

After discharge, one trained investigator in each center will follow up all patients at 40 weeks PMA and at 12, 26, 52, and 104 weeks corrected age. We will provide free neurodevelopmental impairment examinations to promote follow-up rates. For potential dropouts during the follow-up, we will expand the sample size by $20 \%$ and the missing data will be ignored in the analysis. The outcomes at each time point mentioned above will be collected by the investigators (Table 1).

\section{Data management}

All documents and source documents will be stored in the data center. These documents include informed consent, all reports, administrative forms, copies of medical records, and other identifiable patient material. In the data center, data will be stored in file cabinets locked to areas with limited access, thus keeping participants anonymous. All electronic participant data will be deposited in our department's relevant platform. The principal investigator will have access to the final data sets and be responsible for the data. After application and permission, related researchers can have access to the anonymous data for future studies. Additionally, we will deliver a completely deidentified data set to an appropriate data archive for sharing purposes.

\section{Statistical analysis}

The analyses will be based on the intention-to-treat (ITT) principle in accordance with the Consolidated 
Table 1 SPIRIT figure: showing time points for enrollment, interventions, and assessment

\begin{tabular}{|c|c|c|c|c|c|c|c|}
\hline \multirow{3}{*}{ Time point } & \multicolumn{7}{|l|}{ Study period } \\
\hline & \multirow{2}{*}{$\begin{array}{l}\text { Enrollment } \\
\text { At } 4 \text { weeks } \\
\text { after birth }\end{array}$} & \multirow{2}{*}{$\begin{array}{l}\text { Allocation } \\
\text { At } 4 \text { weeks } \\
\text { after birth }\end{array}$} & \multicolumn{4}{|l|}{ Post-allocation } & \multirow{2}{*}{$\begin{array}{l}\text { Close-out } \\
\text { At } 104 \text { weeks } \\
\text { corrected age }\end{array}$} \\
\hline & & & $\begin{array}{l}\text { At } 36 \text { weeks } \\
\text { postmenstrual } \\
\text { age }\end{array}$ & $\begin{array}{l}\text { At } 40 \text { weeks } \\
\text { postmenstrual } \\
\text { age }\end{array}$ & $\begin{array}{l}\text { At } 12,26 \text { weeks } \\
\text { corrected age }\end{array}$ & $\begin{array}{l}\text { At } 52 \text { weeks } \\
\text { corrected age }\end{array}$ & \\
\hline \multicolumn{8}{|l|}{ Enrollment: } \\
\hline Eligibility screen & $x$ & & & & & & \\
\hline Informed consent & $x$ & & & & & & \\
\hline Demographic data & $x$ & & & & & & \\
\hline Allocation & & $x$ & & & & & \\
\hline \multicolumn{8}{|l|}{ Intervention: } \\
\hline Hydrocortisone & & $x$ & & & & & \\
\hline Placebo & & $x$ & & & & & \\
\hline \multicolumn{8}{|l|}{ Assessments: } \\
\hline Baseline variables assessments & $x$ & $x$ & & & & & \\
\hline Primary outcome & & & $x$ & & & & \\
\hline \multicolumn{8}{|l|}{ Secondary outcomes: } \\
\hline Brain volume & & & & $x$ & & & \\
\hline Others & & & $x$ & & & & \\
\hline \multicolumn{8}{|l|}{ Long-term outcomes: } \\
\hline $\begin{array}{l}\text { Neurodevelopmental } \\
\text { impairment, hearing loss, and } \\
\text { blindness }\end{array}$ & & & & & & & $x$ \\
\hline Number of readmissions & & & & & & $x$ & \\
\hline $\begin{array}{l}\text { Weight, length, and head } \\
\text { circumference }\end{array}$ & & & & $x$ & $x$ & $x$ & $x$ \\
\hline $\begin{array}{l}\text { Behavioral problems, premature } \\
\text { birth-related mortality }\end{array}$ & & & & & & & $x$ \\
\hline
\end{tabular}

Standards of Reporting Trials (CONSORT). Statistical analyses will be performed with SAS version 9.2 (SAS Institute, Cary, North Carolina). Data will be presented as the mean \pm standard deviation or median and interquartile range depending on their distribution. Categorical data, including the primary outcome, will be analyzed using the chi-square test between two groups. Continuous data will be analyzed using Student's $t$ test or the Mann-Whitney test as appropriate. The effect of $\mathrm{HC}$ on the dichotomous outcomes will be assessed by multivariable logistic regression analysis including possible confounders, including birth weight and gestational age. Statistical results will be reported as point estimates and 95\% confidence intervals.

Mid-term data analysis will be conducted after inclusion of the first 98 patients. The principal investigator will decide whether any measure should be taken in light of the results. Case report forms (CRFs) will be entered by two independent researchers to ensure accuracy.

\section{Ethical and academic management}

The research protocol was reviewed and approved by the Ethics Committee of Southwest Medical University, and we will not begin recruiting at other centers in the trial until local ethical approval has been obtained. The ethics committee will be responsible for supervising all procedures of the study. Prior to making any changes to the study protocol, a written application will be submitted to the ethics committee for approval, and new written informed consent will be obtained from the guardians.

An independent academic board, which is composed of competent department, data monitoring, and safety management advisors, has been implemented to oversee unwanted effects and risks, as well as the collection and management of the data. The academic board will review all research data every 3 months. Until the included patients are discharged, each potential adverse effect will be monitored and reported to the academic board within $48 \mathrm{~h}$. All members of the academic board are independent of the sponsor and trial investigators and free of competing interests. 
Participants, as well as the researchers, evaluators, doctors, and nurses who are responsible for screening, recruitment, dispensing medicine, and outcome assessment, will remain blinded to treatment allocations. The researchers conducting the statistical analysis will know only the group codes but not the corresponding interventions. When severe adverse effects occur, the academic board has the right to unseal emergency envelopes. The reason for unblinding and time will be recorded. We prepared an insurance policy for every participant. This will provide ancillary and posttrial care in the case of any harm resulting from participation in the clinical trial.

After consulting the academic board, the treatment will be suspended for the following conditions: (1) serious adverse reactions (death or severe infections) that occur during the testing or (2) a guardian request withdraw their infant from the study at any time and without any consequences. The primary outcome and other data collected for participants until the date of withdrawal will be used and analyzed as part of the ITT population.

Patients and the public were not involved in the design of the present study. The results of the trial will be published in a peer-reviewed journal and will be presented at national and international conferences. The final author's confirmation will be based on the contribution to the study and there are no plans to utilize professional writers.

\section{Quality control}

To ensure a full understanding and mastery of the standard operating procedures, all researchers will be trained before the trial begins. Independent investigators at the Affiliated Hospital of Southwest Medical University will regularly monitor the quality of the data collected on the CRFs. If any error exists, researchers will be notified immediately.

\section{Discussion}

In babies $<26^{+0}$ weeks gestational age, several problems, such as PDA, severe apnea, and high thorax compliance, may lead to noninvasive respiratory dependency at day 28 without any link to BPD. Furthermore, a higher rate of sepsis was observed in infants born at 24-25 weeks gestational age who were treated with hydrocortisone in a previous study [21]. Therefore, we will restrict the eligible patients to those between $26^{+0}$ and $30^{+6}$ weeks gestational age.

Because inflammation plays a key role in BPD, postnatal glucocorticoids are thought to be the most effective therapeutic strategy for preterm infants with a high risk for developing BPD [35], but they are accompanied by severe longterm adverse neurodevelopmental outcomes $[11,12]$. These adverse outcomes seem to be associated with the timing of steroid exposure and the cumulative dosage of glucocorticoids; the earlier the treatment initiation and/or the larger the dosage, the higher the risk of adverse outcomes $[11,15]$.

Following the actual recommendations, many infants presenting with early BPD do not receive any anti-inflammatory therapy, and a high percentage of premature infants are still oxygen-dependent or on respiratory support at 36 weeks PMA (moderate or severe BPD) [27-29], which is associated with poor long-term pulmonary outcomes, including home oxygen therapy and a high rate of bronchopulmonary infections and rehospitalization [2]. A recent study showed that moderate or severe BPD at 36 weeks PMA was associated with increased mortality in the first year of life and moderate to severe neurodevelopmental impairment (55\%) [6]. Thus, we hypothesize that anti-inflammatory treatment of infants meeting the NICHD criteria for BPD at 28 days of life may decrease the incidence of moderate or severe BPD at 36 weeks PMA and reduce long-term adverse outcomes.

In previous studies, early low-dose HC therapy in ventilator-dependent infants improved the rate of survival without BPD at 36 weeks PMA [21] without adverse neurodevelopmental outcomes at 2 years of age $[22,24]$. Thus, it seems reasonable to use low-dose $\mathrm{HC}$ to treat infants with early BPD to improve pulmonary outcome. Accordingly, the efficacy and safety of this treatment must be proven by a randomized controlled trial.

\section{Trial status}

Data collection will begin from August 1, 2019, and will end in July 31, 2022. The protocol version number and date are as follows: Second Edition, 13 March, 2019.

\section{Abbreviations \\ BPD: Bronchopulmonary dysplasia; PND: Postnatal day; NICHD: National Institute of Child Health and Human Development; PMA: Postmenstrual age; GA: Gestational age; HC: Hydrocortisone; RCT: Randomized controlled trial; GCP: Good Clinical Practice; GMP: Good Manufacturing Practice; IVH: Intraventricular hemorrhage; PVL: Periventricular leucomalacia; NEC: Neonatal necrotizing enterocolitis; ROP: Retinopathy of prematurity; CPAP: Continuous positive airway pressure; ITT: Intention to treat; CONSORT: Consolidated Standards of Reporting Trials; CRFs: Case report forms}

\section{Acknowledgements \\ We appreciate the efforts of all research staff participating in this trial. We also acknowledge the helpful support from all participants.}

\section{Authors' contributions}

$\mathrm{XL}$ and $\mathrm{CW}$ conceived the idea for the project and contributed to the design of the study. YH drafted the first manuscript. $X \mathrm{~L}$ is the guarantor and get the funding. All authors $(\mathrm{YH}, \mathrm{YZ}, \mathrm{SG}, \mathrm{XW}, \mathrm{NH}, \mathrm{DZ}, \mathrm{WD}, \mathrm{XL}$ and $\mathrm{CW}$ ) read, provided feedback, and approved the final manuscript.

\section{Funding}

This work was supported by the Research Foundation of Southwest Medical University (http://xmgl.swmu.edu.cn/) with project number 2015SX-0056. These funding and supporting sources have no role in the design of the study and collection, analysis, and interpretation of data or in writing the manuscript. 


\section{Availability of data and materials}

The authors declare that all relevant data will be included in the article or supplementary files. Additional data are available from the corresponding author on reasonable request.

\section{Ethics approval and consent to participate}

Central ethical approval has been confirmed from the Southwest Medical University (ref approval no. 20190425-2), and we will not begin recruiting at other centers in the trial until local ethical approval has been obtained. All participants will receive written informed consent from the guardian.

\section{Consent for publication}

Not applicable.

\section{Competing interests}

The authors declare that they have no competing interests.

\section{Author details}

'Department of Neonatology, Affiliated Hospital of Southwest Medical University, 25 Taiping Road, Luzhou 646000, Sichuan, China. ${ }^{2}$ Department of Neonatology, Sichuan Provincial Hospital for Women and Children, Chengdu, Sichuan, China. ${ }^{3}$ Department of Neonatology, Chengdu Women's and Children's Central Hospital, Chengdu, Sichuan, China. ${ }^{4}$ Birth Defects Clinical Medical Research Center of Sichuan Province, Luzhou, Sichuan, China. ${ }^{5}$ Department of Perinatology, Affiliated Hospital of Southwest Medical University, Luzhou, Sichuan, China. ${ }^{6}$ Department of Neonatology, Klinikum Aschaffenburg, Am Hasenkopf 1, Aschaffenburg, 63739 Aschaffenburg, Bavaria, Germany.

Received: 7 January 2020 Accepted: 20 August 2020 Published online: 03 September 2020

\section{References}

1. Stoll BJ, Hansen NI, Bell EF, Eunice Kennedy Shriver National Institute of Child Health and Human Development Neonatal Research Network, et al. Trends in care practices, morbidity, and mortality of extremely preterm neonates, 1993-2012. JAMA. 2015:314:1039-51.

2. $\quad$ Cheong Jeanie LY, Doyle LW. An update on pulmonary and neurodevelopmental outcomes of bronchopulmonary dysplasia. Semin Perinatol. 2018;42:478-84

3. Stoecklin B, Simpson SJ, Pillow JJ. Bronchopulmonary dysplasia: rationale for a pathophysiological rather than treatment based approach to diagnosis. Paediatr Respir Rev. 2018;32:91-7.

4. Jobe $\mathrm{AH}$, Bancalari E. Bronchopulmonary dysplasia. Am J Respir Crit Care Med. 2001;163:1723-9.

5. Bancalari E, Jain D. Bronchopulmonary dysplasia: can we agree on a definition? Am J Perinatol. 2018;35(6):537-40. https://doi.org/10.1055/s-00381637761.

6. Jensen EA, Dysart K, Gantz MG. The diagnosis of bronchopulmonary dysplasia in very preterm infants: an evidence-based approach. Am J Respir Crit Care Med. 2019. https://doi.org/10.1164/rccm.201812-23480C.

7. Network VO. 2018 manual of operations: part 2. Data definitions \& infant data forms. 2017. Available online at: https://public.vtoxford.org/wpcontent/uploads/2017/04/Manual_of_Operations_Part2_v22-1.pdf

8. Higgins RD, Jobe AH, Koso-Thomas M, et al. Bronchopulmonary dysplasia: executive summary of a workshop. J Pediatr. 2018;197:300-8.

9. Shaffer ML, Baud O, Lacaze-Masmonteil T, et al. Effect of prophylaxis for early adrenal insufficiency using low-dose hydrocortisone in very preterm infants: an individual patient data meta-analysis. J Pediatr. 2019; 207:136-142.e5

10. Shinwell ES, Eventov-Friedman S. Impact of perinatal corticosteroids on neuromotor development and outcome: review of the literature and new meta-analysis. Semin Fetal Neonatal Med. 2009;14(3):164-70.

11. Doyle LW, Cheong JL, Ehrenkranz RA, Halliday HL. Early (<8 days) systemic postnatal corticosteroids for prevention of bronchopulmonary dysplasia in preterm infants. Cochrane Database Syst Rev. 2017;10:CD001146.

12. Doyle LW, Cheong JL, Ehrenkranz RA, Halliday HL. Late ( $>7$ days) systemic postnatal corticosteroids for prevention of bronchopulmonary dysplasia in preterm infants. Cochrane Database Syst Rev. 2017;10:CD001145.

13. Halliday Henry L. Update on postnatal steroids. Neonatology. 2017;111: 415-22.
14. Committee on Fetus and Newborn. Postnatal corticosteroids to treat or prevent chronic lung disease in preterm infants. Pediatrics. 2002;109(2): 330-8.

15. Watterberg KL, American Academy of Pediatrics Committee on Fetus and Newborn. Policy statement-postnatal corticosteroids to prevent or treat bronchopulmonary dysplasia. Pediatrics. 2010;126(4):800-8.

16. Huang CC, Lin HR, Liang YC, Hsu KS. Effects of neonatal corticosteroid treatment on hippocampal synaptic function. Pediatr Res. 2007;62:267-27.

17. Hitzert MM, Benders Manon JNL, Roescher AM, et al. Hydrocortisone vs. dexamethasone treatment for bronchopulmonary dysplasia and their effects on general movements in preterm infants. Pediatr Res. 2012;71:100-6.

18. Watterberg KL, Gerdes JS, Gifford KL, et al. Prophylaxis against early adrenal insufficiency to prevent chronic lung disease in premature infants. Pediatrics. 1999;104:1258-63.

19. Watterberg KL, Gerdes JS, Cole CH, et al. Prophylaxis of early adrenal insufficiency to prevent bronchopulmonary dysplasia: a multicenter trial. Pediatrics. 2004;114:1649-57.

20. Peltoniemi $\mathrm{O}$, Kari MA, Heinonen $\mathrm{K}$, et al. Pretreatment cortisol values may predict responses to hydrocortisone administration for the prevention of bronchopulmonary dysplasia in high-risk infants. J Pediatr. 2005;146:632-7.

21. Baud O, Maury L, Lebail F, et al. Effect of early low-dose hydrocortisone on survival without bronchopulmonary dysplasia in extremely preterm infants (PREMILOC): a double-blind, placebo-controlled, multicentre, randomised trial. Lancet. 2016:387:1827-36.

22. Baud O, Trousson C, Biran V, et al. Association between early low-dose hydrocortisone therapy in extremely preterm neonates and neurodevelopmental outcomes at 2 years of age. JAMA. 2017:317:1329-37.

23. Watterberg KL, Shaffer ML, Mishefske MJ, et al. Growth and neurodevelopmental outcomes after early low-dose hydrocortisone treatment in extremely low birth weight infants. Pediatrics. 2007;120:40-8.

24. Baud O, Trousson C, Biran V, et al. Two-year neurodevelopmental outcomes of extremely preterm infants treated with early hydrocortisone: treatment effect according to gestational age at birth. Arch Dis Child Fetal Neonatal Ed. 2019;104:F30-5.

25. Peltoniemi OM, Lano A, Puosi R, et al. Trial of early neonatal hydrocortisone: two-year follow-up. Neonatology. 2009;95:240-7.

26. Onland W, Cools F, Kroon A, et al. Effect of hydrocortisone therapy initiated 7 to 14 days after birth on mortality or bronchopulmonary dysplasia among very preterm infants receiving mechanical ventilation: a randomized clinical trial. JAMA. 2019;321:354-63.

27. Gomez PE, Concina VA, Samide A, et al. Bronchopulmonary dysplasia: comparison between the two most used diagnostic criteria. Front Pediatr. 2018;6:397.

28. Ehrenkranz RA, Walsh MC, Vohr BR, et al. Validation of the National Institutes of Health consensus definition of bronchopulmonary dysplasia. Pediatrics. 2005:116(6):1353-60.

29. Kair LR, Leonard DT, Anderson JM. Bronchopulmonary dysplasia. Pediatr Rev 2012:33(6):255-63 quiz 263-4.

30. Watterberg K. Evidence-based neonatal pharmacotherapy: postnatal corticosteroids. Clin Perinatol. 2012;39(1):47-59.

31. Hellström-Westas $L$, Rosén I, Continuous brain-function monitoring: state of the art in clinical practice.[J] .Semin Fetal Neonatal Med, 2006, 11: 503-511.

32. Ment LR, Bada HS, Barnes P, Grant PE. Practice parameter: neuroimaging of the neonate: report of the Quality Standards Subcommittee of the American Academy of Neurology and the Practice Committee of the Child Neurology Society. Neurology. 2002;58:1726-38.

33. Neu J, Walker WA. Necrotizing enterocolitis. N Engl J Med. 2011:364:255-64.

34. International Committee for the Classification of Retinopathy of Prematurity. The international classification of retinopathy of prematurity revisited. Arch Ophthalmol. 2005;123:991-9.

35. Cheong JLY, Doyle LW. Long-term effects of postnatal corticosteroids to prevent or treat bronchopulmonary dysplasia: balancing the risks and benefits. Semin Fetal Neonatal Med. 2019;24:197-201.

\section{Publisher's Note}

Springer Nature remains neutral with regard to jurisdictional claims in published maps and institutional affiliations. 